\title{
La ciencia de la ciudadanía: más allá de la necesidad de expertos*
}

\author{
STEVE FULLER \\ Universidad de Warwick
}

Resumen. Comienzo examinando algunas pistas, en gran medida falsas, que se han seguido desde los griegos para definir la naturaleza de la ciudadanía científica en una democracia. Sin embargo, el linaje que va desde Platón al positivismo proporciona un contexto útil para entender la evolución de la concepción moderna de conocimiento experto y de los diferentes problemas que éste plantea a las democracias modernas. Estos problemas giran en torno a las cuestiones de la institucionalización - en concreto, a cómo diseñar instituciones que respeten la idea de conocimiento como un principio de orden social sin caer en el gobierno del conocimiento experto-. Después de analizar dos recientes propuestas alemanas que siguen estas líneas, defiendo la institucionalización de las «conferencias de consenso», o jurados de ciudadanos. Finalmente, considero alguna de las implicaciones epistemológicas sociales más generales del papel del «ciudadano científico».
AbSTRACT. I begin by examining some largely false leads from the Greeks in defining the nature of scientific citizenship in a democracy. Nevertheless, the lineage from Plato to positivism does provide a context for understanding the evolution of the modern conception of expertise and the distinct problems it poses to modern democracies. These problems revolve around issues of institutionalisation - specifically, how to design institutions that respect the idea of knowledge as a principle of social order without succumbing to rule by expertise-. After analysing two recent German proposals along these lines, I defend the institutionalisation of «consensus conferences», or citizen juries. Finally, I consider some the more general social epistemological implications of the role of «citizen scientist».

\section{Introducción: El peligro de buscar precedentes históricos en los griegos}

La Atenas clásica introdujo una influyente división tripartita del trabajo social: algunos problemas debían ser debatidos en público, otros decididos en privado, y otros delegados a los expertos. Sin embargo, estas tres divisiones no se corresponden con la organización natural del trabajo social en las democracias constitucionales modernas. Por otra parte, las distinciones griegas originales proporcionan una base efectiva para la crítica de las moder-

\footnotetext{
* Traducción de Inés Gutiérrez González y Amalia Vijande Martínez (Universidad de Oviedo).
} 
nas. Hoy en día entendemos que «lo público» es «político» en el sentido estrecho de «basado en intereses», mientras que los atenienses entendían que era «político» en el sentido amplio de una res publica - una cosa que sólo existe en público, esto es, algo que afecta a todo el mundo por igual (Fuller, 2000a, cap. 1) - . Tendemos a considerar «lo privado» como el ámbito de la conciencia y la conducta personal, mientras que los atenienses lo asociaban con la gestión del hogar (el significado original de «economía»), la única plaza en la que las mujeres eran iguales a los hombres. La «privacidad» en este sentido se manifestaba en la condición de la hacienda de cada uno y en su relación con esclavos y vecinos. Aunque no sería erróneo decir que tanto los antiguos como los modernos usan la vida privada de alguien como una medida de adecuación para la vida pública, sería una grave equivocación no tomar en cuenta el cambio en los significados de «público» y «privado» a lo largo de los últimos 2.500 años.

Pero los atenienses entendieron «conocimiento experto» en sentido restringido, como una técnica que requiere entrenamiento especializado, y éste es quizás el conjunto de connotaciones vinculado a este concepto que puede, potencialmente, llevar a más equívocos. El caso paradigmático de conocimiento experto era una destreza que alcanzaba un fin dentro de un conjunto de medios estrechamente limitado - como cuando un escultor talla un busto de mármol-. A oídos modernos, esto suena a ciencia normal kuhniana (o, para el caso, a política profesional en las democracias constitucionales, de acuerdo con Weber y Schumpeter), en la que un experto ejerce la autoridad final sobre un dominio de la realidad, que es inherentemente tan compleja que para su dominio debe ser dividida (Zolo, 1992). Sin embargo, para los griegos, la necesidad de «entrenamiento especializado» surgía menos de la complejidad de la cuestión que de una estricta división del trabajo entre medios y fines, tal que el cliente proporcionaba los fines y al «experto» se le confiaba sólo su realización dentro de un medio específico (Fuller, 2000b: cap. 2, núm. 70). El experto griego era, por tanto, menos un agente autónomo que una tecnología humanoide - en el mejor de los casos era un comerciante, en el peor un esclavo-. A este respecto, los expertos griegos no eran tan diferentes de los investigadores contratados por «obra y servicio» que pueblan cada vez más la academia. La calidad de su trabajo se determina sólo por su habilidad para satisfacer a sus clientes, independientemente de los fines de éstos. La responsabilidad sobre los fines recae enteramente en el cliente, y el experto todo lo más tiene el poder de retirar sus servicios si no está de acuerdo con ellos.

El tipo de gente que hoy llamamos «expertos», especialmente los científicos cuya autoridad se busca en los foros de diseño de políticas y en los judiciales, no existía en la Atenas clásica. Desde el punto de vista griego, serían entidades mestizas que combinan la especialización del técnico y la autonomía del ciudadano, con la excepción de que los expertos de hoy care- 
cen de las restricciones impuestas a ambos tipos de personas en el mundo antiguo. En Atenas, los clientes y los ciudadanos tenían el poder y la motivación para actuar como controles externos. Por el contrario, los expertos de hoy hablan con autoridad sobre parte de la realidad sin tales controles con excepción de los que proceden de otros expertos-, que tienen pocos incentivos para hablar en contra de un colega, y aún menos para contribuir a la devaluación general del conocimiento experto, tal como ocurre a menudo hoy en los procesos judiciales acusatorios (punto sobre el que volveré en la próxima sección).

Como he dicho antes, los griegos incluían en los asuntos públicos cualquier tema que concerniera globalmente a todos - esto es, era necesario que tuviera relevancia universal-. Esto incluiría a la ciencia tal como se entendía clásicamente, algo que se podría mantener incluso en nuestros días. Aristóteles señaló, célebremente, al principio de su Metafísica, que sólo era normal para los ciudadanos volverse hacia los problemas de relevancia universal cuando hubieran satisfecho las cuestiones relativas a su subsistencia material. La ciencia y la política eran por lo tanto vocaciones gemelas para el tiempo libre. Desde ese punto de vista, los antagonistas sofistas de Sócrates eran serpientes en este Jardín del Edén deliberativo. Sostenían que las artes de la deliberación pública podían ser enseñadas como habilidades especiales que ofrecían resultados fiables. En algún sentido, los sofistas eran como Weber, Schumpeter, y otros proveedores de «política profesional». No obstante, Sócrates se resistió a sus interlocutores sofistas, no porque el poder se pudiera concentrar en las manos de expertos especialmente entrenados, sino por la razón más fundamental de que dudaba que las habilidades prometidas por los sofistas pudieran realmente poseerse. Para Sócrates, la competencia en los asuntos públicos simplemente se derivaba de la educación liberal normalmente proporcionada a las élites, que solamente recibían entrenamiento sobre cómo hacer un uso productivo de su ocio (Villa, 2001).

De todas formas, lo que se sigue de este punto no está completamente claro. Por un lado, podría seguirse que la democracia sólo sirve para las élites que cumplan el requisito de una educación liberal, y no puede enseñarse sobre una base ad hoc a cualquiera que pueda satisfacer la tarifa del sofista. Por otro lado, podría seguirse que todo el mundo debe recibir una educación liberal como prerrequisito para la participación plena en la política democrática. En cierto sentido, estas dos opciones podrían no ser tan opuestas - si entendemos que el carácter de la educación liberal debe cambiar para que pueda contribuir a una verdadera ciudadanía democrática-. Por ejemplo, el currículum debería dirigirse a estudiantes que provienen de trasfondos sociales diversos, en cuyo caso entonces la instrucción necesitaría ser más explícita y estandarizada que, por ejemplo, un diálogo socrático en el cual hay un entendimiento común previo de la 
«sabiduría convencional» que el diálogo pone a prueba y desafía. Además, el contexto de instrucción en sí mismo podría necesitar un cambio, de modo que un seminario íntimo se reemplazaría por una gran aula de clase, que se aproxima más al emplazamiento de las grandes asambleas democráticas. De hecho, podría resultar que a medida que más gente se incorporara a la «educación liberal», ésta asumiera naturalmente las características sugeridas por la pedagogía sofística.

En realidad, el entendimiento que tenía de la situación el ateniense medio podría haber sido que Sócrates y los sofistas quizás no fueran tan diferentes. La historia sólo comienza a dibujar una afilada distinción entre aquellos (como Sócrates) genuinamente preocupados por la búsqueda de la verdad y aquellos (como los sofistas) preocupados en apariencia únicamente por ganar la discusión después de que Platón acuñara despectivamente el término «retórica» referido a las pretensiones de la pedagogía sofística. Sin embargo, tras los debates entre Sócrates y los sofistas había una preocupación común que muy raramente se aprecia en las interpretaciones de Platón, pero que explica su diferencia de estrategia. Ambos estaban preocupados por mantener la investigación abierta. Si con algo estaban obsesionados los atenienses, era con la novedad, y por lo tanto generalmente asociaban técnica con rutina y trabajo pesado. Mientras los medios y los fines de la investigación no se distinguieran claramente - pensaba Sócrates -, la gente se vería continuamente obligada a pensar sobre estas cuestiones en relación unas con otras. No habría una lógica predispuesta, puesto que los fines de uno podrían cambiar al oír lo que el otro dice. Por supuesto, los sofistas no objetaban esta orientación general. No obstante, creían que esto podría facilitarse haciendo que las habilidades de la argumentación estuvieran disponibles de forma más general, de modo que más gente pudiera tener la oportunidad de influir sobre la investigación común. En su mayoría extranjeros, los sofistas sentían que Sócrates - un héroe militar ateniense - quería reservar la razón pública para aquellos de «buena naturaleza». Su renuencia a admitir la instrucción formal en las artes democráticas olía a un «nativismo» aristocrático que sólo serviría a horizontes políticos estrechos.

Aquí merece la pena resaltar que el célebre mandamiento sofístico de hacer que el argumento más débil parezca el más fuerte no suponía un desprecio por la verdad. Más bien, reflejaba un escepticismo subyacente que decía que puesto que nunca se puede conocer la verdad última, el hecho de que un argumento ahora parezca más fuerte o más débil siempre será una cuestión de contingencia, por ejemplo, de quién ha tenido el tiempo y el dinero para recoger las evidencias correctas para el momento en que el argumento deba ser defendido. Lo que parece falso ahora puede aún volverse verdadero en el futuro ( $\mathrm{y}$ viceversa), dada una apropiada redistribución de recursos. En realidad, no se debería considerar que los sofistas se burlaran de la búsqueda de la verdad, sino que más bien promovían una ética existencialista 
del discurso donde uno asume la responsabilidad de construir las situaciones de modo que se capacite al público para responder de tantas formas como sea posible. Los contextos comunicativos en nuestro propio tiempo que más reclaman este tipo de tratamiento son precisamente aquellos que ponen en cuestión la identidad de los expertos apropiados: e. g., allí donde la medicina convencional requiere justificación frente a la medicina alternativa; donde la biología evolutiva requiere defensa contra los teóricos del diseño inteligente, etc. La idea aquí es recuperar, sobre bases normativas relativas a la promoción de la democracia, la naturaleza abierta e inquisitiva de la argumentación que era característica del foro ateniense. Nada de esto niega la relevancia del conocimiento técnico experto en las deliberaciones públicas. Más bien se trata de obligar a los representantes de estas formas de conocimiento a poner a prueba sus argumentos fuera de los foros auto-certificadores asociados con los procesos de «revisión por pares» (para una defensa teórica y práctica de esta posición «neosofística», véase Fuller y Collier, 2003; veáse también Fuller, 2002, Apéndice, para una discusión de la «revisión por pares extendida»).

\section{El surgimiento de los expertos en la política democrática: de Platón al positivismo}

El inestable ambiente político creado por los encuentros dialécticos públicos de Sócrates y los otros sofistas que instruyeron a los líderes de su juventud afectó profundamente a Platón. Después de la caída de Atenas ante Esparta en las Guerras del Peloponeso, optó por restringir la dialéctica a un escenario enclaustrado, que sólo debía abrirse a la sociedad más amplia una vez que los iniciados estaban suficientemente seguros, por su propio juicio y conocimiento, de que las demandas populares no iban a influir sobre ellos en exceso. En términos de la historia intelectual occidental, el positivismo modula la motivación filosófica original de Platón a través de una versión secularizada de la historia de la salvación cristiana, en la cual Newton funciona como la figura de Cristo. Esto recoge tanto el espíritu del proyecto original de Augusto Comte como sus efectos residuales en el positivismo lógico del siglo Xx, que se liberó del claro historicismo del proyecto de Comte al tiempo que mantuvo la fijación en Newton como modelo de lo que significa expresarse a uno mismo científicamente, y una vaga creencia en que el conocimiento científico más excelso será el que proporcione la salvación.

El problema conceptual central del positivismo ha sido definir una vanguardia científica capaz tanto de ofrecer una guía para los no iniciados como de cambiar ella misma a la luz de posteriores evidencias e ideas. Donde Platón había deseado producir implacables reyes filósofos que gobernaran como monarcas absolutos, los positivistas han imaginado un gobierno más 
diferenciado pero no menos autorizado (¿autoritario?) de expertos, cada uno oligarca sobre su propio dominio de conocimiento. A este respecto, el positivismo es el compañero de viaje filosófico de la burocracia.

No resulta sorprendente que la relación del positivismo con la democracia haya tenido altibajos. Como Platón, los positivistas han temido más que a nada al desacuerdo público prolongado, y por lo tanto han tendido a demonizarlo como «irracional» y «no cognitivo». Su concepto de autoridad «plural» supone competencias que no se superponen, como, por ejemplo, que las cuestiones legislativas se reducen a judiciales en lo relativo al conocimiento experto al que cada una debe someterse. Esto está en agudo contraste con la mayor parte de las concepciones de la democracia republicana cívica, que basan la representación en grupos de interés, cada uno investido con una competencia apropiada a la promoción de sus intereses, pero que generalmente tratan sobre cuestiones en las que también otros grupos pueden tener competencia. Puesto que las cuestiones de política pública afectan típicamente a varios de estos grupos a la vez, las decisiones colectivas no consisten en la identificación de un grupo adecuado cuyo juicio debe prevalecer. Más bien se espera que el debate público en sí mismo lleve a una solución que trascienda los puntos de partida de todos los grupos, pero que no obstante logre servir a sus respectivos intereses.

Así, hay una ambigüedad fundamental en la apelación del positivismo a una razón organizada, o «ciencia», en la esfera pública. Algunas veces esta ambigüedad se soluciona diplomáticamente afirmando que los positivistas consideran la ciencia como la principal fuente de unidad política. Como mínimo, esto implica que está en el interés de todos los miembros de la sociedad perseguir sus fines por medios científicos, puesto que ello les permitirá economizar esfuerzos y por lo tanto disponer de más tiempo para disfrutar los frutos de su trabajo. Ernst Mach es quien más se acercó a la defensa de esta posición en su forma pura. Encaja cómodamente con la idea libertaria de que los regímenes democráticos deben posibilitar el máximo desarrollo de las capacidades de cada uno.

De cualquier forma, muchos positivistas han extraído una conclusión más que puede frustrar este impulso libertario. Desde Comte en adelante, ha sido común argumentar que la ciencia puede unificar la política resolviendo, conteniendo o sorteando el conflicto social. Aquí se supone que un procedimiento bien establecido o un conjunto decisivo de hechos reemplazan formas más «primitivas» y cambiantes de resolución de conflictos, como la guerra e incluso a veces el debate abierto - que supuestamente comprometen la integridad de los puntos de vista opuestos en función de la conveniencia-. De acuerdo con esto, una política científica no debe solamente satisfacer a las partes implicadas: debe llegar a la solución «correcta».

Seguramente, incluso esta mentalidad admite una interpretación democrática, puesto que investigadores sociales positivistas han estado entre los 
primeros en presentar «datos» de partes cuyas voces no era probable que fueran escuchadas en una asamblea abierta. Típicamente, esto ha ocurrido en encuestas diseñadas para representar el rango empírico completo de una población objetivo. No obstante, queda abierta la pregunta de quién es exactamente quien recoge los beneficios políticos de estas voces recientemente articuladas: ¿la población investigada, los investigadores mismos, o los clientes de los investigadores? Más aún, una vez que una población objetivo ha sido empíricamente registrada, ¿siguen siendo sus miembros «objetos de investigación», o se les promueve a la categoría de investigadores de pleno derecho capaces de desafiar los hallazgos y métodos de los investigadores originales? Probablemente el tratamiento más sofisticado de estas cuestiones en el contexto de la investigación sobre política social inspirada por el positivismo en Estados Unidos se encuentra en Campbell (1988).

Estas delicadas cuestiones surgen porque en última instancia el positivismo vuelve a Platón de su lado convirtiendo una jerarquía estática en un orden temporal. Donde Platón imaginó que la autoridad manaba hacia abajo desde el rey filósofo, en una estructura social basada en castas, los positivistas han imaginado que toda la humanidad debe pasar (en una proporción variable) a través de una secuencia de estados - teológico, metafísico y científico - que reconstruye el viaje socio-epistémico desde la esclavitud a la autonomía. En la utopía positivista, es posible que todo el mundo sea un experto en un dominio específico. Más aún, hay una receta para la conversión del platonismo en positivismo. Procede aislando un dominio de investigación de las contingencias que rodean sus manifestaciones, de modo que su naturaleza esencial pueda ser desentrañada. Mientras Platón reservaba tal investigación a los reyes filósofos, los positivistas se han inclinado más a menudo por cuerpos profesionales autorizados por el Estado. Y en lugar de la intuición intelectual de Platón (nous), los positivistas han tratado de ganar acceso epistémico por medios comparativos históricos y experimentales. Ha habido tanto un problema práctico como teórico con la conversión positivista de la aristocracia epistémica de Platón en el clero (o «clerecía») laico de los expertos.

El problema práctico se relaciona con el uso de «testigos expertos» en procedimientos judiciales, especialmente en los sistemas legales basados en la acusación (i. e., investigaciones dirigidas por los adversarios), más que en la inquisición (i.e., investigaciones dirigidas por jueces). El papel social del testigo experto fue inventado por Hugo Munsterberg, a quien William James invitó desde Alemania en 1897 para fundar el laboratorio psicológico de Harvard. Munsterberg era lo que ahora podríamos llamar un axiólogo «naturalista», discípulo del neokantiano Heinrich Rickert. Es decir, sostenía (como Mill, James y Freud) que el valor está determinado por el deseo - esto es, la evidencia de que un objeto es considerado valioso (por alguien) debe preceder a las atribuciones de valor para tal objeto-. A su llegada a 
los Estados Unidos, Munsterberg reetiquetó su perspectiva como «psicología aplicada», un campo capaz de adaptar a la gente tanto para el empleo como para el castigo, en virtud de su habilidad para identificar las capacidades e inclinaciones de las personas. Pero Munsterberg, acostumbrado al sistema legal inquisitivo de Alemania, no pudo anticipar que el sistema acusatorio de América impediría a los jueces regular el uso de los testigos expertos. Así, se hizo posible -y se podría mantener que ahora rutinarioque ambos adversarios en un juicio movilizaran a testigos expertos a su servicio, que resultaban mutuamente contradictorios. El crítico del Derecho Peter Huber (1991) ha denunciado esta tendencia como «ciencia basura» (junk science), instando a los jueces a emplear estándares más exigentes para la admisión de testimonio científico. De todas formas, esto puede ser simplemente equivalente a delegar en los jueces el poder de resolver indeterminaciones inherentes en la aplicabilidad de hallazgos científicos a las decisiones legales (Jasanoff, 1995). En cualquier caso, un sistema legal acusatorio claramente disminuye el poder de los expertos para resolver disputas sociales.

Pero, en última instancia, hay límites teóricos a la gobernabilidad por el conocimiento experto. Consideremos el trabajo de Otto Neurath, el principal organizador del Círculo de Viena, que engendró el positivismo lógico como la esperanzada síntesis dialéctica del autoritarismo comtiano y el libertarismo de Mach - y al que llamó, en condiciones poco favorables, «marxismo neutral» (Proctor 1991, cap. 9)-. Un ejemplo del enfoque notablemente ambiguo de Neurath sobre la planificación social fue su intento de aislar la esencia de la «economía de guerra» de modo que su mecanismo de planificación central eficiente pudiera ser transferido a ambientes donde pudiera tener consecuencias socialmente más saludables. Aquí Neurath anticipaba lo que Alvin Gouldner (1970) consideró como dos caras de la misma moneda: el «estado de bienestar» y el «estado de guerra», puesto que la misma estructura organizacional (en este caso, una concentración de recursos en el Estado-nación), podía tener consecuencias radicalmente diferentes, dependiendo del entorno político sustentante. No obstante, como muchos críticos de Neurath han señalado, el positivismo parece haber heredado la ingenuidad política del platonismo, que confunde el hecho de que, por ejemplo, la «economía de guerra» pueda ser identificada analíticamente como una característica de muchas sociedades, con la habilidad del analista para transferirla a nuevos entornos sociales un proceso que puede requerir la imposición de una fuerza considerable para mantener todos los demás factores ambientales constantes. Si algo merece el nombre de «falacia positivista», es este ejemplo de ingenuidad, que asimila demasiado fácilmente el foro con el laboratorio, sin considerar los costes de motivar al pueblo a ajustarse al ideal. 


\section{La necesidad de instituciones portadoras de conocimiento para contrarrestar el dominio del conocimiento experto}

El platonismo y el positivismo comparten una intuición general de que el conocimiento debe ser el principio de la estratificación social. Esta intuición es común a la mayoría de la literatura sobre epistemología social. Mi posición difiere al mantener que esta intuición no puede ser válida sin la adecuada atención al diseño de las instituciones portadoras de conocimiento (Fuller, 2002, cap. 4). En esta sección consideraré dos propuestas alemanas recientes, pero bastante divergentes, en esta línea - la primera de Gernot Böhme, la segunda de Wolfgang van den Daele-. Ambos tratan de recuperar un elemento de democracia ante la invasión del conocimiento experto de lo que ambos llamarían el «mundo de la vida». Merece la pena notar que, en el pasado, ambos ocuparon un lugar prominente dentro del denominado movimiento de finalización, que en los setenta del siglo XX unió una concepción autopoietica del cambio científico (inspirada por Thomas Kuhn) con una iniciativa de investigación estratégica dirigida por el Estado para las ciencias «maduras» (una traducción inglesa de los principales artículos de este movimiento es Schaefer, 1984). Hoy en día Böhme y Van den Daele parecerían enfatizar, respectivamente, el primer y último estado de la teoría de la finalización original.

Böhme ha defendido que la comunidad científica debe mirar a su propia historia para encontrar una versión éticamente más adecuada y ecológicamente más consciente de sí misma, asentada quizás en la visión holística del mundo de la Naturphilosophie del siglo XIX. A este respecto, la desconfianza pública contemporánea en la ciencia es sintomática de la autoalienación de la propia ciencia. Si bien hay mucho que elogiar en la perspectiva de Böhme, deja abierta la cuestión de la institucionalización. Presumiblemente, el plan es más profundo que una simple exhortación a los científicos maduros a bucear en las obras de Goethe en busca de inspiración.

Uno puede imaginar al menos dos posibles implicaciones políticas que cambiarían radicalmente la cara de la producción de conocimiento. La primera sería requerir que los estudiantes de ciencia estudiaran las historias de sus campos, de modo que aprendieran acerca de marcos teóricos alternativos para moldear sus investigaciones antes de que les adoctrinaran en el marco dominante. La segunda sería involucrar un rango más amplio de científicos practicantes en el gobierno de sus propios campos, puesto que es bien sabido que cuanto más distantes se ven los propios científicos a sí mismos de la investigación específica de vanguardia, más abiertos están a preocupaciones interdisciplinarias y extradisciplinarias (también llamadas transdisciplinarias). De cualquier forma, ambas implicaciones políticas encontrarían fuerte resistencia desde el establishment científico por lo que los seguidores de 
Mary Douglas en sociología del conocimiento científico reconocerían como «transgresiones de las coordenadas y de grupo» (group-grid violations) (por ejemplo, Bloor, 1983). Por un lado, los científicos tendrían que abrir sus puertas a enfoques opuestos a aquellos en los que sus identidades profesionales se forjaron originalmente (i.e., una transgresión de grupo); por otro, tendrían que subvertir la meritocracia de la ciencia, que valora a los investigadores en términos de su cercanía a las fronteras de la investigación especializada (i.e., una transgresión de coordenadas).

Pero quizá todo esto muestra que los problemas que enfrenta la relación de la ciencia con la sociedad en general no se solucionarán a través de nuevas políticas de autogobierno. En ese caso, tenemos la propuesta de Van den Daele de que las aplicaciones de la ciencia deben estar sujetas a control externo, especialmente para no interferir con la promoción de conocimientos alternativos, también denominados conocimientos indígenas. Esta propuesta presupone que se pueden trazar distinciones bastante claras entre la ciencia y otras formas de conocimiento, así como entre la investigación y sus aplicaciones. Estas presuposiciones deberían recordar el problema de la sociedad del conocimiento descrito anteriormente y la ambigüedad interpretativa de sus implicaciones políticas exactas: ¿debemos comprometernos con la promoción de ciertas formas de conocimiento, sin importar quiénes son los portadores del conocimiento, o con la promoción de grupos de personas que mantienen ahora, pero no necesariamente en el futuro, ciertas formas de conocimiento?

En resumen, la propuesta de Van den Daele no se enfrenta directamente al desafío de la Ilustración que supone que la gente mejorará su destino en la vida cambiando su fidelidad hacia formas tradicionales de conocimiento por otras formas más científicas. En otras palabras, del hecho de que el conocimiento esté históricamente atado a un grupo con intereses particulares no se sigue que tal conocimiento siga siendo indefinidamente relevante para promocionar sus vidas. Pero, por supuesto, puede merecer la pena cultivar ciertas formas de conocimiento indígena para complementar, suavizar o revisar el conocimiento científico. Pero la cuestión de si los más adecuados para realizar esta tarea son los portadores de conocimiento indígenas es una cuestión abierta. Una buena referencia sobre estas cuestiones es la práctica de lingüistas interesados en preservar lenguajes minoritarios frente a la invasión generalizada de la lengua inglesa: asegurar un suministro continuo de hablantes nativos no es la única estrategia posible. También se puede alentar a los no nativos para que aprendan las lenguas minoritarias.

Como hemos visto, las propuestas de Böhme y Van den Daele para gobernar el conocimiento subestiman la relevancia normativa de las instituciones. Ambas están primariamente orientadas a articular una visión normativa - la de Böhme de una Naturphilosophie actualizada y la de Van den Daele de un pluralismo cultural forzado por el Estado - que deja abier- 
ta la cuestión de las instituciones concretas que podrían realizarla. De todas formas, si uno está interesado en que estos resultados se produzcan de forma recurrente, y no simplemente a través de intervenciones ad hoc, entonces la selección de los vehículos institucionales adecuados es de capital importancia. Las soluciones normativas a los problemas de legitimidad institucional pueden parecer autoritarias si se presta atención exclusiva a la articulación de los fines de la producción de conocimiento, asumiendo de este modo que cualquier medio está virtualmente justificado en el proceso de adquirir tales fines.

Más aún, incluso cuando los marcos institucionales están pensados seriamente, algunas veces los elegidos no logran producir un todo mayor que la suma de las partes originales. En ese sentido, las instituciones pueden reflejar las presuposiciones sociológicas de sus diseñadores sin habilitar a sus participantes para lograr más de lo que ellos mismos podrían hacer si se les dejara con sus propios mecanismos. Se pueden encontrar ejemplos en los muy publicitados paneles de «evaluación de tecnologías» de Van den Daele, que unen a los representantes de los principales grupos de interés para diseñar líneas políticas para cuestiones tan controvertidas como los alimentos genéticamente modificados (Van den Daele, Pühler, y Sukopp, 1997). A menudo se llega a un punto muerto, que se utiliza después para justificar el recurso a un panel ético consultivo de carácter gubernamental para ocuparse de las diferencias de valores irresolubles en situaciones concretas. El problema aquí, sugiero, no es que los diferentes grupos de interés tengan visiones del mundo radicalmente diferentes, sino más bien que los paneles de evaluación de tecnologías están diseñados para reforzar, no para sublimar, esas diferencias. Así, el pluralismo cultural se convierte en la profecía que se cumple a sí misma.

Para un contraste institucional, consideremos las «conferencias de consenso», que he defendido como vehículos para involucrar al público en el diseño de políticas de ciencia y tecnología (Fuller, 2002, cap. 4). Esta configuración restringe el papel de los interesados a testigos en un juicio, y los jurados son miembros del público que no están afiliados formalmente con ninguno de los interesados. Por supuesto, los ciudadanos jurado pueden entrar ya en los procesos con fuertes puntos de vista, pero sus medios de vida no se verán directamente afectados por la perspectiva de cambiar sus opiniones a lo largo de la conferencia de consenso. La presuposición sociológica aquí es que, en una cuestión política dada cualquiera, la mayoría de la gente no está tan clara y fuertemente alineada con una particular visión del mundo como para ser impermeable a la discusión. La criticada «ignorancia» del público sobre cuestiones de ciencia y tecnología es sólo una debilidad si se traduce en cerrazón de mente. Pero la apelación a un amplio rango de representantes de grupos de interés como testigos en las conferencias de consenso está especialmente diseñada para revelar la naturaleza en gran 
medida disputada del conocimiento que el público supuestamente no tiene, lo que después fuerza a los participantes de la conferencia a estar más vigilantes y abiertos, además de animarles a convencerse de que ellos pueden «marcar la diferencia».

Por supuesto, nada de esto garantiza que las soluciones particulares de un jurado de ciudadanos satisfagan completamente a los interesados. Pero aquí la legitimidad institucional de la conferencia de consenso deberá prevalecer sobre las objeciones a sus decisiones particulares. Para contar como una mejora sobre los paneles de interesados, los resultados de la conferencia de consenso deben ser vinculantes para la legislación sobre ciencia y tecnología, permitiendo al mismo tiempo la revisión e incluso la revocación de la legislación en el futuro, a la luz de sus consecuencias. Merece la pena señalar que puesto que éstos son desiderata normativos generales para el diseño de las instituciones, suscitan cuestiones sobre la deseabilidad última de, por ejemplo, mantener fuertes distinciones entre los conocimientos científicos e indígenas si los portadores de conocimiento relevantes cambian sus orientaciones epistémicas tras su participación en conferencias de consenso. De hecho, la mayoría de lo que se diagnostica como un antagonismo hacia la ciencia puede en realidad ser una alienación del proceso de construcción de políticas científicas, que a lo largo del tiempo puede reificarse, con la ayuda de intelectuales y otros constructores de mitos, hacia una identidad epistémica «positiva» alternativa, lo que se denomina una comunidad imaginada (Anderson, 1983). Las conferencias de consenso están bien situadas para invertir esa tendencia. Pero hay obstáculos prácticos, que se explorarán en la siguiente sección.

\section{Problemas en la institucionalización de las conferencias de consenso}

Éste es un momento interesante en la «comprensión pública de la ciencia». Por un lado, nunca antes habían sido tan populares los libros y programas de televisión sobre ciencia. Por otro, las matrículas en cursos sobre temas científicos y la confianza pública en la ciencia parecen estar en declive. Los científicos naturales insisten en que el público lego debería aprender más ciencia o remitirse a las opiniones de las autoridades científicas relevantes. Los científicos sociales observan que a menudo los propios científicos están en desacuerdo entre ellos, y el público no experto con frecuencia sabe cosas que los científicos no saben.

¿Qué es exactamente lo que sabe el público lego que podría ser relevante, por ejemplo, para el «Managing Radiactive Waste Safely» («Gestión Segura los Residuos Radioactivos»), para citar el título de un documento de consulta pública que el Departamento de Medio Ambiente del Reino Unido hizo circular en Internet en 2001? La respuesta más general, y quizás la más 
obvia, es que la gente conoce la relación entre lo que creen y lo que consideran factible y deseable. Cuando la gente se opone a una solución «científicamente bien fundada» de un problema social, no se debe por lo general a una ignorancia de cuestiones científicas, ni tampoco a una falta de respeto por el conocimiento experto. Se debe más bien a que no sienten que su experiencia personal y su capacidad para emitir juicios independientes haya sido tomada en consideración de manera seria en los procesos políticos.

Así, a los lectores minuciosos de los documentos de consulta pública se les podría perdonar cierto escepticismo acerca del interés del gobierno británico en una participación pública sustantiva. Consideremos esta declaración de intenciones, tomada del sumario ejecutivo:

Queremos inspirar confianza pública en las decisiones y en la manera en que se ponen en práctica. Para hacer esto, tenemos que demostrar que todas las opiniones se tienen en consideración; que las elecciones entre ellas se hacen de manera clara y lógica; que los valores y las preocupaciones de la población están enteramente reflejadas en este proceso; y que la información que se proporciona es clara, precisa, imparcial y completa. Así, nos proponemos crear un cuerpo fuerte, independiente y autorizado que nos asesore sobre qué información se dispone, qué información complementaria es necesaria, y cuándo ha sido recogida la información suficiente para tomar decisiones sobre cómo se deberían gestionar los residuos radioactivos del Reino Unido [...]. Después de esto, podemos abrir un debate acerca de dónde se deberían depositar estos residuos a largo plazo.

En los capítulos 5 y 6, podemos ver que el «cuerpo independiente y autorizado» se elegirá entre expertos en gestión de residuos nucleares bien consolidados tanto en la academia como en la industria. Ese cuerpo definirá el conocimiento base y el marco de opciones disponibles para que se consideren en no menos de 15 formas de «participación pública», abarcando desde foros de Internet y encuestas telefónicas hasta grupos de discusión y conferencias de consenso.

Hay al menos dos razones importantes para pensar que este procedimiento no «inspirará confianza pública». La primera es que el público no está implicado en la construcción del conocimiento base que necesita para considerar o tener en cuenta las distintas opciones de gestión de los residuos. Parece que se le impide al público examinar seriamente, por ejemplo, cómo los científicos llegan a concluir que una cierta opción provoca un nivel específico de riesgo. En vez de eso, al público se le promete una rigurosa, aunque unificada, exposición de conocimiento experto sobre el que basar sus deliberaciones. Presumiblemente cualquier desacuerdo existente entre los expertos será resuelto, o al menos minimizado, antes de que el público delibere. El público no estará en posición de investigar, y aún menos de cuestionar, las diferencias entre las interpretaciones de expertos y público lego sobre la gestión de residuos nucleares. 
La segunda razón es que el documento incluye casi todas las formas de consulta pública sin comprometerse previamente a acatar los resultados de ninguna de ellas. El resultado es una versión democrática de la estrategia maquiavélica del «divide y vencerás». Cuando al público lego se le da manga ancha para expresar sus opiniones, es comprensible que el resultado sea una cacofonía. Sin un procedimiento para dirigir los múltiple puntos de vista hacia un resultado claro, como un voto específico, los líderes políticos pueden seleccionar y mezclar a discreción las visiones en el paquete político más conveniente. De hecho, dadas suficientes formas de «participación pública», el gobierno no debería tener problemas para reclamar algún tipo de respaldo público para cualquier política que adopte.

La idea de que diferentes tipos de contribuciones posibilitan mejores tomas de decisiones tiene sentido sólo si los métodos usados para obtener los datos proporcionan diferentes accesos relevantes a la población, y hay algún procedimiento claro para integrar esas diversas contribuciones. Sin estas condiciones, simplemente nos quedamos con un montón de datos ruidosos que pueden ser interpretados como se quiera. La mala ciencia social sencillamente se convierte en una tapadera para las «políticas de siempre». Este problema es peor aún en los casos en los que están implicadas la ciencia y la tecnología porque no está claro lo que podría significar «representar» la opinión pública en esas materias. La ciencia y la tecnología tienden a tener un impacto generalizado sobre todos los sectores de la sociedad, sin embargo el público por lo general no tiene ninguna visión clara sobre ello. Así, sorprende poco que sean los grupos con los puntos de vista más claros - los «interesados»- los que con frecuencia terminan definiendo los términos de la representación. La solución a este problema no son técnicas más ingeniosas para sondear la opinión pública, sino nuevas instituciones de diseño de decisiones políticas que permitan al público en general desarrollar sus opiniones sobre ciencia y tecnología. El genio histórico de los regímenes democráticos descansa sobre instituciones que construyen el todo político como algo más, y no menos, que la suma de sus partes ciudadanas. La gran virtud de las conferencias de consenso es que su estructura de jurado asegura que las opiniones del público no están dominadas por los que se declaran a sí mismos interesados.

Las conferencias de consenso algunas veces también reciben el nombre del «jurados de ciudadanos» o, de manera más ambiciosa, «experimentos en democracia deliberativa». Por lo general una conferencia de consenso tiene dos fases. En la primera, a unos 10 o 15 miembros del público se les atribuye el poder de tomar declaración a varios expertos y grupos de interés. Como en un juicio, no hay un acuerdo previo entre los testigos para presentar ante el jurado un enfoque uniforme. De hecho, se anima al jurado de ciudadanos a investigar las discrepancias en el testimonio experto. En la segunda fase, los miembros del jurado reflexionan entre ellos para llegar a unas 
directrices políticas que permitan un legislación sobre tema. Los propios miembros del jurado trazan las directrices, como en una asamblea constitucional. Los resultados se trasladan entonces al cuerpo legislativo electo, como estaba previsto.

Una conferencia de consenso se reúne, por lo general, durante unos tres o cuatro días, aunque ese tiempo puede dividirse en fines de semana consecutivos, dadas las otras obligaciones en la vida de los ciudadanos que forman el jurado, y también para animarles a discutir de manera informal en Internet. Además, podría necesitarse una investigación previa en grupos de discusión para asegurar que la competencia de las conferencias de consenso no es ni demasiado amplia ni demasiado restringida. El Instituto Galés para la Salud y el Bienestar Social (Welsh Institute for Health and Social Care) ha estimado el coste de su reciente conferencia de consenso sobre pruebas genéticas para trastornos médicos comunes en unas 20.000 libras esterlinas.

Las conferencias de consenso surgen en Alemania y en los Estados Unidos a finales de los años sesenta del siglo Xx. En un principio tenían como objetivo delegar poder en las comunidades locales sobre asuntos o materias que les concernían directamente (e. g., planificaciones urbanísticas), y demostrar que el público no experto podía tomar decisiones políticas, al menos de manera tan acertada como sus representantes electos. Sin embargo, desde mediados de los ochenta, las conferencias de consenso han desempeñado una tercera función que surge, en gran medida, en respuesta al aumento de la relevancia de los temas biomédicos y medioambientales para la política pública. Han sido foros para la integración de cuerpos de conocimiento experto y lego en lo que puede ser descrito como «disputas jurisdiccionales»: ¿quién sabe más sobre cómo funciona mi cuerpo y mi mundo - los médicos, los científicos del Estado o yo mismo-?

El Instituto Loka de Richard Sclove en Amherst, Massachussets, lleva un registro de las conferencias de consenso que han sido convocadas en todo el mundo: alrededor de 50 desde 1985. Se han celebrado conferencias de consenso en Australia, Austria, Canadá, Dinamarca, Francia, Alemania, Israel, Japón, Holanda, Nueva Zelanda, Noruega, Corea del Sur, Suiza, Reino Unido y Estados Unidos. Entre los temas tratados se incluyen la clonación, los alimentos genéticamente modificados, la terapia génica y otras investigaciones genéticas en general, la «sociedad de la información», y la gestión de residuos nucleares. En uno de los países, Dinamarca, las conferencias de consenso se convocan regularmente siempre que el parlamento está considerando alguna legislación relacionada con ciencia y tecnología. La legislación resultante se justifica conformándose a las directrices marcadas en la conferencia de consenso.

Los expertos, sistemáticamente, se quedan impresionados por la seriedad con la que los miembros de las conferencias de consenso asumen las tareas que se les asignan, y especialmente por la fuerza argumentativa de las direc- 
trices políticas resultantes. Seguramente, muchos ciudadanos-jurados poseen al principio fuertes opiniones sobre, por ejemplo, el uso apropiado de la investigación genética. Sin embargo, al final de la conferencia son capaces de distinguir entre lo que aceptarían para ellos mismos y sus familias y lo que permitirían hacer a otros. Puede que ellos personalmente nunca deseen recurrir a la terapia génica, pero ahora son capaces de entender por qué otras personas podrían hacerlo.

Reconocer la diferencia entre los intereses privados y los públicos es crucial para la política científica en una sociedad liberal. Siempre habrá gente cuyas convicciones les impidan estar de acuerdo con la investigación biomédica o con tratamientos médicos que, por ejemplo, pudieran introducir sustancias animales en el cuerpo humano. La pregunta es si permitirían a aquellos que mantienen convicciones diferentes actuar de otro modo. Las conferencias de consenso son crisoles donde se forja un sentido común de las diferencias permisibles, de las que en última instancia toda la sociedad puede aprender.

Sin este reconocimiento, no está claro cómo las innovaciones científicas pueden conseguir apoyo democrático generalizado. Todas las innovaciones se originan como una disidencia minoritaria que tiene como objetivo cambiar la norma. Pero si la voz de todo el mundo se escucha de igual manera, entonces, o bien la mayoría elimina el disenso o bien, si no hay una mayoría clara, la política menos ofensiva o más segura recoge la mayoría de los votos. Las conferencias de consenso están diseñadas específicamente para contrarrestar la tendencia de las democracias a asociar todas las ideas nuevas con el intento por parte de un grupo de interés de obtener ventaja sobre el resto.

Las conferencias de consenso no deberían confundirse con un «pleno municipal» en el que aquellos que tienen intereses fuertes, tanto a favor como en contra de una política propuesta, dominan la discusión y determinan los resultados. Más bien, como en una selección ordinaria de los miembros de un jurado, aquellos que tienen poder para tomar decisiones políticas se separan de las partes interesadas, cuya única función es ofrecer testimonio. Aunque los ciudadanos que forman el jurado con frecuencia tienen opiniones personales fuertes, éstas serán diversas y no necesariamente conectadas con sus trabajos diarios. Tales son las condiciones necesarias para una deliberación colectiva. El hecho de que las conferencias de consenso se hayan ensayado en tantos países - incluyendo algunos en los que se carece de tradiciones democráticas fuertes - sugiere que la ciencia no es todavía una «cosa pública», una res publica. Las llamadas campañas de «comprensión pública de la ciencia» han tenido éxito al fomentar el interés en la ciencia sin proporcionar necesariamente salidas para expresar y aplicar ese interés. La respuesta polarizada de los Estados Unidos y Europa a la introducción de alimentos genéticamente modificados ilustra este problema. Sin 
algo parecido a las conferencias de consenso para focalizar el pensamiento y la acción colectiva, la gente reacciona bien como consumidores pasivos, bien como militantes activistas. Ninguna de las opciones constituye un adecuado compromiso público con la ciencia.

Por otra parte, a pesar de sus impresionantes resultados, las conferencias de consenso no han recibido apoyo incondicional por parte de las comunidades de científicos naturales ni sociales. Los científicos biomédicos, en particular, con frecuencia se muestran incómodos con la tendencia de las conferencias de consenso a desdibujar la distinción entre el desarrollo y la aplicación de una investigación científica. Mantienen que el público debería determinar cómo se usa la investigación, pero no cómo se lleva a cabo en la práctica. El argumento se basa tanto en los miedos de los científicos respecto a su propia autonomía, como en las preocupaciones sobre el estado del conocimiento lego de la ciencia. Sin embargo, la línea que divide el desarrollo de investigación y la aplicación está más clara en la teoría que en la práctica. Cuando una conferencia de consenso considera un asunto como la terapia génica o los alimentos genéticamente modificados, son muchos los temas que afloran y que difuminan la división: ¿cómo se van a probar los tratamientos o los productos antes de que puedan adquirirse de manera general?, ¿qué grupos son los que más probablemente obtendrán ventaja o desventaja del desarrollo de esos tratamientos o productos? Para responder estas preguntas, los ciudadanos del jurado tomarán por supuesto testimonio a los expertos más relevantes.

Los científicos sociales suscitan una crítica algo más sutil. Afirman que la ciencia tiene «múltiples públicos», cada uno con su propio conjunto de intereses. Además, el estado de la investigación científica misma está sujeto a múltiples interpretaciones y a una considerable incertidumbre. No se puede esperar que una conferencia de consenso resuelva todas esas diferencias en una «percepción pública de la ciencia» común. Una perspectiva histórica es útil para responder a esta objeción. El avance de la democracia ha estado siempre perseguido por el escepticismo sobre la capacidad de gente con puntos de vista radicalmente diferentes para prosperar bajo un marco de gobierno común. Para tales escépticos, una democracia podría existir sólo entre aquellos que tienen ideas afines, no entre aquellos cuyos intereses están en constante tensión. Así, los sociólogos que estudian la ciencia hoy tienden a privilegiar las formas «locales» de conocimiento cultivadas por comunidades particulares sobre los intentos - por ejemplo, por parte del Estado o de la industria - de imponer una percepción específicamente «científica» de las cosas. Podría parecer que las conferencias de consenso proporcionan incluso una oportunidad más para favorecer este tipo de imposición.

En última instancia, la respuesta a esta objeción es que el registro de legislaciones y constituciones es mucho más positivo de como lo presentan los escépticos. La conferencia de consenso es simplemente el último capítu- 
lo en la historia de la gestión democrática del conflicto. Además, dado que la ciencia se ha convertido en algo esencial en la vida de todo el mundo, ahora más que nunca necesita incorporarse dentro de los mecanismos ordinarios de gobierno. Más allá de insistir simplemente en la «incertidumbre» del conocimiento científico, las conferencias de consenso pueden proporcionar las bases para lo que las legislaturas hacen mejor, a saber, desarrollar experimentos sociales corregibles, sólo que esta vez sobre la ciencia misma.

\section{Conclusión: convertirse en un ciudadano científico hoy}

Antes de Irwin (1995), la expresión «ciudadano-científico» se refería normalmente al ideal ilustrado ejemplificado por los padres fundadores de los Estados Unidos, Benjamín Franklin y Thomas Jefferson, que hicieron contribuciones a la ciencia y a la tecnología a la vez que promovían innovaciones políticas. Como los atenienses clásicos, asociaban las dos actividades como expresiones naturales de su libertad (quizás el más perdurable, y todavía incomprendido, legado de esta sensibilidad es la concepción de autonomía de Kant que supone un sentido de auto-determinación que refleja la determinación divina del cosmos). Las colonias británicas americanas propiciaron un ambiente especialmente acogedor para la reinvención de la virtud cívica republicana en la segunda mitad del siglo XVIII - especialmente cuando se las comparaba con la propia Gran Bretaña.

Un buen ejemplo es la carrera del químico filosófico y cristiano inconformista Joseph Priestley, cuya «Sociedad Lunar» de Birmingham reunía a mecánicos e ideólogos de una forma que la Royal Society había prohibido claramente en sus estatutos (Uglow, 2002). Priestley se vio obligado a emigrar a los Estados Unidos después de haber apoyado públicamente la Revolución Francesa de 1789, cuya causa (creía él) se basaba en una percepción científica más adecuada de la naturaleza humana que la de sus oponentes. (Un buen ejemplo de lo que Priestley tenía en mente es que Jeremy Bentham, poco después, afirmó haber derivado de él el eslogan utilitarista «el mayor bien para el mayor número».) Por supuesto, hoy Priestley es recordado principalmente como la persona que no logró ver que su descubrimiento del «aire desflogisticado» equivalía al oxígeno, el elemento que encabezó la Revolución Química. Da la casualidad de que la nemesis de Priestley, Antoine Lavoisier, fue guillotinado durante el Terror por defender un sentido del continuismo político análogo al igualmente fatal continuismo científico de Priestley. La diferencia, por supuesto, es que la «fatalidad» de Lavoisier le costó su vida, mientras que la de Priestley le costó la reputación.

Hoy en día la «ciencia del ciudadano» pude referirse al menos a dos sentidos diferentes de la producción democrática de conocimiento que están en desacuerdo el uno con el otro. Por un lado, la ciencia del ciudadano puede 
ser diseñada para dar crédito a una forma de «conocimiento local» a pesar de su falta de credenciales científicas. Esto es deseable en tanto que expresión de una identidad local y como correctivo metodológico para las formas más abstractas y generales de conocimiento a las que se dedican las investigaciones científicas profesionales. La mayor parte del trabajo en los estudios sociales de la ciencia relacionado con la «comprensión pública de la ciencia» (e. g., Irwin y Wynne, 1996) podría caer bajo esa categoría. Aquí se podría pensar en el ciudadano científico como alguien que rechaza potencialmente esquemas a gran escala por contradecir la experiencia local. En cualquier caso, estaría claro que el objetivo político de la ciencia del ciudadano es mantener un cierto nivel de autonomía política. Por otro lado, una función sutilmente diferente de la ciencia del ciudadano es extender y profundizar en el sufragio democrático ordinario dando voz a un grupo de personas tradicionalmente excluido de las decisiones políticas, en las que la ciencia juega un papel cada vez más relevante. Pero como en el caso de las conferencias de consenso, es más importante que la gente marginada participe en las decisiones a que sus opiniones permanezcan intactas hasta el final del proceso. De hecho, en la medida en que las conferencias de consenso son un crisol para forjar una sociedad más democrática, se espera que todas las partes modifiquen sus opiniones durante las deliberaciones. De esto no se sigue necesariamente que alcancen un juicio unánime $-\mathrm{y}$, a este respecto, «conferencia de consenso» puede ser un nombre inapropiado-. Pero este sentido de ciencia del ciudadano implica que el mero hecho de que una forma de conocimiento sea bien «local», bien «científica», no la acredita intrínsecamente en el escenario político. Más bien, el foro es él mismo un escenario para la producción de conocimiento original.

Finalmente, permítasenos meditar sobre el papel de la epistemología en el régimen democrático de la ciencia del ciudadano. Las teorías clásicas del conocimiento - aquellas obsesionadas con la adquisición de «creencia verdadera justificada»- presuponen un agente que trata el conocimiento como un fin aislable al que se le debería dar prioridad sobre el resto de fines. Podríamos llamar a esto el punto de vista del participante desinteresado. Por el contrario, los posmodernos tienden a adoptar el punto de vista del situado, i. e., del participante interesado. La orientación normativa de mi epistemología social es, en líneas generales, la del no-participante interesado en el sistema de conocimiento. Por lo general considero el conocimiento como un medio para otros fines humanos (que pueden ser ellos mismos epistémicos), pero la participación de alguien en el proceso de conocimiento normalmente se reduce al meta-nivel de la investigación, que es el diseño y evaluación de los regímenes de producción de conocimiento que otros llevan a cabo. Estos regímenes abarcan temas de gestión fiscal y de personal, de responsabilidad social, así como, de manera específica, formas de control de calidad basadas en procesos y productos. Así, mi antiguo interés en presentar la epistemolo- 
gía social como equivalente a «política del conocimiento» (Fuller, 1998, Apéndice C) y «gestión del conocimiento» (Fuller, 2002) está fundado en la idea de que, hablando en términos generales, los que prescriben y los que evalúan (los legisladores y los gestores) de la producción del conocimiento no son los mismos - en términos de identidades o intereses - que los productores de conocimiento de primer orden («trabajadores»).

Ha habido dos modelos históricos de no-participación interesada en un esfuerzo colectivo: uno antiguo y elitista, el otro moderno y populista. Hemos empezamos con una discusión sobre el primer modelo, sacado de la antigua Atenas, que está basado en el cliente que proporciona orientación al artesano, que a su vez posee la técnica necesaria para implementar las ideas del cliente. En Atenas, el cliente recibía el crédito del éxito del producto, el artesano el de su fracaso. El segundo modelo, sacado de las elecciones democráticas modernas, se basa en que los ciudadanos seleccionan regularmente a los políticos que consideran que mejor proporcionarán una orientación política coherente para sus diversos intereses. Aquí la distribución de mérito y culpa es menos clara, excepto por una tendencia a atribuir el éxito a la constitución global del sistema de gobierno (a menudo al contrato social original, en el caso de los Estados Unidos), y el fracaso a los individuos particulares (ciudadanos o políticos). Esta tendencia encaja con la relación entre la confianza global en la ciencia y la falta de confianza en las teorías científicas particulares que marca la ética falsacionista asociada con Karl Popper (Fuller, 2003). Si debemos vivir en un mundo que no permite elecciones regulares sobre ciencia, las conferencias de consenso ofrecen el medio más factible para realizar el ideal popperiano (pero para pistas sobre una visión alternativa véase Fuller, 2002, parte III).

\section{REFERENCIAS}

ANDERSON, Benedict (1983): Imagined Communities, Londres, Verso.

BLOOR, David (1983): Wittgenstein: A Social Theory of Knowledge, Oxford, Blackwell.

CAMPBell, Donald (1988): Methodology and Epistemology for the Social Sciences, Chicago, University of Chicago Press.

DAele, Wolfgang van den; PÜHLER, Alfred y SuKopP, Herbert (1997): Transgenic Herbicide-Resistant Crops: A participatory technology assessment, Wissenschaftszentrum Berlin für Sozialforschung, Discussion paper, FS II, pp. 97-302.

FULLER, Steve (1988): Social Epistemology, Bloomington, Indiana University Press.

- (2000a): The Governance of Science, Milton Keynes, UK, Open University Press.

- (2000b): Thomas Kuhn: A Philosophical History for Our Times, Chicago, University of Chicago Press.

- (2002): Knowledge Management Foundations, Woburn, Mass., ButterworthHeinemann. 
- (2003): Kuhn vs. Popper, The Struggle for the Soul of Science, Cambridge, UK, Iconbooks.

- y Collier, James H. (2003): Philosophy, Rhetoric and the End of Knowledge, 2. ${ }^{\text {a }}$ edn. (Orig. 1993) Hillsdale NJ, Lawrence Erlbaum Associates.

Gouldner, Alvin (1970): The Coming Crisis in Western Sociology, Nueva York, Basic Books.

Huber, Peter (1991): Galileo's Revenge: Junk Science in the Courtroom, Nueva York, Basic Books.

IRWIN, Alan, (1995): Citizen Science, Londres, Routledge

IRWIN, Alan, y BRIAN Wynne (eds.), (1996): Misunderstanding Science? The Public Reconstruction of Science and Technology, Cambridge, UK, Cambridge University Press.

JASANOFF, Sheila (1995): Science at the Bar: Law, Science and Technology in America, Cambridge, MA, Harvard University Press.

Proctor, Robert (1991): Value-Free Science? Cambridge, MA, Harvard University Press.

ScHAEFER, Wolf (ed.) (1984): Finalization in Science, Dordrecht, Neth., Kluwer Academic Press.

Uglow, Jenny (2002): The Lunar Men: The Friends Who Made the Future, Londres, Faber and Faber.

VILla, Dana (2001): Socratic Citizenship, Princeton, Princeton University Press.

Zolo, Daniel (1992): Democracy and Complexity, Cambridge, UK, Polity Press. 